\title{
Stability of degenerate stationary solution to the outflow problem for full Navier-Stokes equations
}

\author{
Yazhou Chen,$^{*} \quad$ Hakho Hong,$^{\dagger} \quad$ Xiaoding Shi ${ }^{\ddagger}$
}

\begin{abstract}
This paper is concerned with the large-time behavior of solutions to the outflow problem of full compressible Navier-Stokes equations in the half line. This is one of the series of papers by the authors on the stability of nonlinear waves to the outflow problem. We show the time asymptotic stability of degenerate (transonic) stationary solution for the general gas including ideal polytropic gas. The key point of the proof is based the new property of the degenerate stationary solution and the delicate energy estimates.
\end{abstract}

MSC 2010: 35Q30, 35B35, 35L65, 76D33, 74J40.

Keywords: compressible Navier-Stokes equations, outflow problem, stationary solution, stability.

\section{Introduction}

The one-dimensional compressible Navier-Stokes equations are described in Eulerian coordinate by the system

$$
\left\{\begin{array}{l}
\rho_{t}+(\rho u)_{x}=0, \quad x>0, \quad t>0 \\
(\rho u)_{t}+\left(\rho u^{2}+p\right)_{x}=\mu u_{x x}, \\
{\left[\rho\left(e+\frac{u^{2}}{2}\right)\right]_{t}+\left[\rho u\left(e+\frac{u^{2}}{2}\right)+p u\right]_{x}=\kappa \theta_{x x}+\mu\left(u u_{x}\right)_{x},}
\end{array}\right.
$$

where $u(x, t)$ is the velocity, $\rho(x, t)>0$ is the density, $\theta(x, t)>0$ is the absolute temperature, $p=p(\rho, \theta)$ is the pressure and $e=e(\rho, \theta)$ is the internal energy, while $\mu$ and $\kappa$ denote the viscosity and the heat-conductivity respectively. Throughout this paper, the pressure $p(\rho, \theta)$ and the internal energy $e(\rho, \theta)$ are assumed to satisfy

$$
p_{\rho}(\rho, \theta)>0, \quad e_{\theta}(\rho, \theta)>0 .
$$

\footnotetext{
*Department of Mathematics, School of Science, Beijing University of Chemical Technology, Beijing 100029, P R China (chenyz@mail.buct.edu.cn).

${ }^{\dagger}$ Institute of Mathematics, State Academy of Sciences, Pyongyang, D P R Korea(hhong@amss.ac.cn).

${ }^{\ddagger}$ Corresponding author. Department of Mathematics, School of Science, Beijing University of Chemical Technology, Beijing 100029, P R China (shixd@mail.buct.edu.cn).
} 
We consider the initial-boundary value (IBV) problem to the system (1.1) on $[0, \infty)$ supplemented with the initial data

$$
\left\{\begin{array}{l}
(\rho, u, \theta)(x, 0)=\left(\rho_{0}, u_{0}, \theta_{0}\right)(x), \quad x>0 \\
\lim _{x \rightarrow \infty}(\rho, u, \theta)(x, t)=\left(\rho_{+}, u_{+}, \theta_{+}\right), \quad t \geq 0
\end{array}\right.
$$

and the boundary condition

$$
u(0, t)=u_{-}<0, \quad \theta(0, t)=\theta_{-},
$$

where $\theta_{ \pm}>0, \rho_{+}>0, u_{ \pm}$are prescribed constants.

Remark 1.1 According to the sign of the velocity $u_{-}(=0,>0,<0)$ on the boundary $x=0$, the following three type of problems are proposed [15]: the impermeable wall problem, the inflow problem and the outflow problem. It should be noted that for the inflow problem, the density $\rho_{-}>0$ must be given on the boundary by the well-posedness theory of the hyperbolic equation $(1.1)_{1}$.

For the Cauchy problem of (1.1), it is well known that the large time behavior of the solutions are described by the corresponding Riemann solutions to the hyperbolic part of the system (1.1) which consist of three basic wave patterns (shock wave, rarefaction wave and contact discontinuity) and their superpositions in the increasing order of characteristic speed. But in the case of the IBV problem of (1.1), not only basic wave patterns but also a stationary solution, which is called the boundary layer solution (BL-solution for brevity), may appear due to the boundary effect. For the IBV problem of isentropic Navier-Stokes equations, Matsumura [15] proposed a criterion on the question when the BL-solution forms and a complete classification about the precise description of the large time behaviors of solutions. Since then, many results have been obtained for the rigorous mathematical justification of this classification. We refer to Matsumura-Mei [16, Matsumura-Nishihara [17] etc. for the impermeable wall problem, to Kawashima-Nishibata-Zhu [12, NakamuraNishibata-Yuge [21, Kawashima-Zhu [13, 14, Huang-Qin [8] etc. for the outflow problem, and to Matsumura-Nishihara [18, Huang-Matsumura-Shi [7], Shi [26], Fan-Liu-Wang-Zhao [3] etc. for the inflow problem and so on. Further, for non-isentropic Navier-Stokes equations (i.e. problem (1.1)), we refer to Huang-Li-Shi [6] etc. for the impermeable wall problem, to Kawashima-Nakamura-Nishibata-Zhu [11, Qin [23], Wan-Wang-Zhao [28, 29], Chen-Hong-Shi [2] etc. for the outflow problems and to Qin-Wang [24, 25], NakamuraNisibata [20], Zheng-Zhang-Zhao [30], Hong-Wang [4, 5] etc. for the inflow problems. More works, please refer to the references therein.

The following focus on the more details for the stability and convergence rate toward stationary solutions to the outflow problem concerned with this paper. For the isentropic Navier-Stokes equations, Kawashima-Nishibata-Zhu [12] first proved asymptotic stability of stationary solutions under small $H^{1}$-initial perturbation. Kawashima-Zhu [13] improved the result in [12] to the combination of stationary solution and rarefaction wave, and HuangQin [8] improved the results in [12, 13] to large initial perturbation. For this stability theorem, a convergence rate was obtained by Nakamura-Nishibata-Yuge [21] by assuming that an initial perturbation belongs to the weighted Sobolev space. Nakamura-Ueda-Kawashima [22] gave a refinement of the stability result established in [21] for a degenerate stationary 
solution. Precisely they obtained a convergence rate under a more moderate assumption rather than the result in [21]. The generalization of these one-dimensional problem to a multi-dimensional half space was also studied, i.e. Kagei-Kawashima [9] proved the asymptotic stability of a planar stationary solution, and Nakamura-Nishibata [19] obtained the convergence rate. For full Navier-Stokes equations (1.1) of ideal polytropic gas, KawashimaNakamura-Nishibata-Zhu [11] first proved the existence, the asymptotic stability and the convergence rate of the stationary solution. Qin [23] proved that the non-degenerate (supersonic or subsonic) stationary solution is asymptotically stable under partially large initial perturbation with the technical condition that the adiabatic exponent $\gamma$ is close to 1. Just recently, Wan-Wang-Zou [28] improved the result in [23] to the large initial perturbation without any restriction on the adiabatic exponent $\gamma$. Also, Wan-Wang-Zhao [29] studied the case when the corresponding time-asymptotic state is a rarefaction wave or its superposition with a non-degenerate stationary solution under large initial perturbation. Just recently, Chen-Hong-Shi [2] studied the stability and convergence rate of a non-degenerate stationary solution to outflow problem in the case of general gas (1.2) including ideal polytropic gas, that is

$$
p=R \rho \theta=A \rho^{\gamma} e^{\frac{\gamma-1}{R} s} \quad \text { and } \quad e=\frac{R}{\gamma-1} \theta,
$$

where $\gamma>1$ is the adiabatic exponent and $A, R$ are both positive constants.

Although considerable progress has been obtained for the stability of stationary solutions to the outflow problem of full Navier-Stokes system (1.1), however most of the results are obtained only in the case of the non-degenerate stationary solution except for Kawashima-Nakamura-Nishibata-Zhu [11] where they proved the stability of degenerate stationary solution for the ideal polytropic gas (1.5), but for the general gas, it is not trivial and the implicit realtions of various thermodynamical variables lead to many complicated terms in the course of establishing the energy estimate.

In this paper, we are interested in the stability of the degenerate (transonic) stationary solution of the outflow problem of (1.1)-(1.4) for the general gases satisfying (1.2) (see Theorem 1.2). The main difficulty for this problem is that the degenerate stationary solution has only the algebraic decay property, not the exponential decay property, therefore the effective methods which used to deal with the non-degenerate (supersonic and subsonic) case (see [23, 28, 29, 2] etc.) are no longer applicable. The key point of the proofs in this paper is to derive the estimates (2.4) in Lemma 2.1, and this is mainly due to our key Proposition 1.1 below which enables us to control the lower order term $I_{i}(i=1,2,3,4,5)$ in Lemma 2.1.

Now we will give some results on the stationary solutions to the outflow problem of full Navier-Stokes system and state our main result. Setting $v=\rho^{-1}$, it is well-known that by using any given two of the five thermodynamical variables $(v, p, e, \theta$ and $s)$, the remaining three variables are smooth functions of them, where $s$ is the entropy of the gas. The second law of thermodynamics $\theta d s=d e+p d v$ asserts that, if we choose $(v, \theta)$ or $(v, s)$ as independent variables and write $(p, e, s)=(p(v, \theta), e(v, \theta), s(v, \theta))$ or $(p, e, \theta)=$ 
$(\widetilde{p}(v, s), \widetilde{e}(v, s), \widetilde{\theta}(v, s))$, respectively, then we can deduce that

$$
\left\{\begin{array}{l}
s_{v}(v, \theta)=p_{\theta}(v, \theta) \\
s_{\theta}(v, \theta)=\frac{e_{\theta}(v, \theta)}{\theta} \\
e_{v}(v, \theta)=\theta p_{\theta}(v, \theta)-p(v, \theta)
\end{array}\right.
$$

or

$$
\begin{cases}\widetilde{e}_{v}(v, s)=-\widetilde{p}(v, s), & \widetilde{e}_{s}(v, s)=\theta \\ \widetilde{p}_{v}(v, s)=p_{v}(v, \theta)-\frac{\theta\left(p_{\theta}(v, \theta)\right)^{2}}{e_{\theta}(v, \theta)}, & \widetilde{p}_{s}(v, s)=\frac{\theta p_{\theta}(v, \theta)}{e_{\theta}(v, \theta)} \\ \widetilde{\theta}_{v}(v, s)=-\frac{\theta p_{\theta}(v, \theta)}{e_{\theta}(v, \theta)}, & \widetilde{\theta}_{s}(v, s)=\frac{\theta}{e_{\theta}(v, \theta)} .\end{cases}
$$

From (1.7) and (1.2) we have

$$
\begin{gathered}
\widetilde{p}_{v}(v, s)=p_{v}(v, \theta)-\frac{\theta\left(p_{\theta}(v, \theta)\right)^{2}}{e_{\theta}(v, \theta)}<0, \\
\left\{\begin{array}{l}
\widetilde{e}_{s s}(v, s)=\frac{\theta}{e_{\theta}(v, \theta)}>0, \quad \widetilde{e}_{v s}(v, s)=-\frac{\theta p_{\theta}(v, \theta)}{e_{\theta}(v, \theta)}, \\
\widetilde{e}_{v v}(v, s)=-p_{v}(v, \theta)+\frac{\theta\left(p_{\theta}(v, \theta)\right)^{2}}{e_{\theta}(v, \theta)}>0,
\end{array}\right.
\end{gathered}
$$

which means that $\widetilde{e}(v, s)$ is convex with respect to $(v, s)$.

Notation: Throughout the rest of this paper, $O(1), c$ or $C$ will be used to denote a generic positive constant independent of $x, t$ and $c_{i}(\cdot, \cdot)$ or $C_{i}(\cdot, \cdot)\left(i \in Z_{+}\right)$stands for some generic constants depending only on the quantities listed in the parentheses. As long as no confusion arises, we denote the usual Sobolev space with norm $\|\cdot\|_{H^{k}}$ by $H^{k}:=H^{k}(0, \infty)$ and $\|\cdot\|_{H^{0}}=\|\cdot\|$ will be used to denote the usual $L_{2}-$ norm.

The stationary solution $(\hat{\rho}, \hat{u}, \hat{\theta})(x)$ of the outflow problem (1.1)-(1.4) must satisfy the following system:

$$
\begin{gathered}
\left\{\begin{array}{l}
(\hat{\rho} \hat{u})_{x}=0, \quad x>0, \\
\left(\hat{\rho} \hat{u}^{2}+\hat{p}\right)_{x}=\mu \hat{u}_{x x}, \\
{\left[\hat{\rho} \hat{u}\left(\hat{e}+\frac{\hat{u}^{2}}{2}\right)+\hat{p} \hat{u}\right]_{x}=\kappa \hat{\theta}_{x x}+\mu\left(\hat{u} \hat{u}_{x}\right)_{x},}
\end{array}\right. \\
(\hat{u}, \hat{\theta})(0)=\left(u_{-}, \theta_{-}\right), \quad \lim _{x \rightarrow \infty}(\hat{\rho}, \hat{u}, \hat{\theta})(x)=\left(\rho_{+}, u_{+}, \theta_{+}\right),
\end{gathered}
$$

where $\hat{p}=p(\hat{\rho}, \hat{\theta}), \quad \hat{e}=e(\hat{\rho}, \hat{\theta})$.

Define the sound speed $c(v, s)$ and the Mach number $M(v, u, s)$ is defined, respectively, by

$$
c(v, s)=\sqrt{\frac{\partial p(\rho, s)}{\partial \rho}}=\sqrt{-v^{2} \widetilde{p}_{v}(v, s)}, \quad M(v, u, s)=\frac{|u|}{c(v, s)},
$$

and set $M_{+}=\frac{\left|u_{+}\right|}{c\left(v_{+}, s_{+}\right)}$, where $s=s(v, \theta)$ and $s_{+}=s\left(v_{+}, \theta_{+}\right)$.

The existence and the properties of the stationary solution $(\hat{\rho}, \hat{u}, \hat{\theta})(x)$ satisfying (1.10) and (1.11) are quoted in the following proposition which is proved by [2]. The proof is given in the Appendix for reader's convenience. 
Proposition 1.1 (Existence of stationary solution). Let $\rho_{+}>0, u_{-}<0, \theta_{ \pm}>0$. The necessary condition for the existence of a solution to the system (1.10), (1.11) is

$$
\hat{\rho} \hat{u}=\rho_{+} u_{+}=\hat{\rho}(0) u_{-}, \forall x>0 .
$$

So, if $u_{+} \geq 0$, there is no solution to the system (1.10), (1.11). If $u_{+}<0$ and (1.2) is hold, then we have the following results.

1) For supersonic case $M_{+}>1$, there exists a positive constant $\delta_{0}$ such that if $\left(u_{-}, \theta_{-}\right) \in$ $\mathcal{M}_{\delta_{0}}:=\left\{(u, \theta) \in R_{-} \times R_{+}||\left(u-u_{+}, \theta-\theta_{+}\right) \mid \leq \delta_{0}\right\}$, then there exists a unique solution $(\hat{\rho}, \hat{u}, \hat{\theta})(x)$ satisfying

$$
\left|\partial_{x}^{k}\left(\hat{\rho}-\rho_{+}, \hat{u}-u_{+}, \hat{\theta}-\theta_{+}\right)\right| \leq C \delta \exp (-\hat{c} x), \quad k=0,1,2,
$$

where $\delta=\left|\left(u_{-}-u_{+}, \theta_{-}-\theta_{+}\right)\right|$and $C, \hat{c}$ are positive constants independent of $x, \delta$.

2) For subsonic case $M_{+}<1$, there exists a positive constant $\delta_{0}$ and a certain subset $\mathcal{M}^{-} \subset \mathcal{M}_{\delta_{0}}$ such that if $\left(u_{-}, \theta_{-}\right) \in \mathcal{M}^{-}$, then there exists a unique solution $(\hat{\rho}, \hat{u}, \hat{\theta})(x)$ satisfying (1.13).

3) For transonic case $M_{+}=1$, under the additional assumption

$$
\begin{aligned}
& p_{\theta}\left(v_{+}, \theta_{+}\right)>0, p_{v v}\left(v_{+}, \theta_{+}\right) \geq 0, p_{\theta \theta}\left(v_{+}, \theta_{+}\right) \geq 0, \\
& p_{v \theta}\left(v_{+}, \theta_{+}\right) \leq 0, e_{v v}\left(v_{+}, \theta_{+}\right) \leq 0, e_{\theta \theta}\left(v_{+}, \theta_{+}\right) \leq 0,
\end{aligned}
$$

there exists a positive constant $\delta_{0}$ and a certain curve $\mathcal{M}^{0} \subset \mathcal{M}_{\delta_{0}}$ such that if $\left(u_{-}, \theta_{-}\right) \in$ $\mathcal{M}^{0}$, then there exists a unique solution $(\hat{\rho}, \hat{u}, \hat{\theta})(x)$ satisfying

$$
\left|\partial_{x}^{k}\left(\hat{\rho}-\rho_{+}, \hat{u}-u_{+}, \hat{\theta}-\theta_{+}\right)\right| \leq \frac{C \delta^{k+1}}{(1+\delta x)^{k+1}}+C \delta \exp (-\hat{c} x), \quad k=0,1,2,
$$

and

$$
\left(\hat{u}_{x}, \hat{\theta}_{x}\right)=\left(a_{1}, a_{2}\right) z^{2}(x)+O\left(z^{3}(x)+\delta \exp (-c x)\right) .
$$

where $a_{i}(i=1,2)$ are positive constants depending only on $\mu, \kappa, \rho_{+}, u_{+}, \theta_{+}$and $z(x)$ is a smooth function satisfying

$$
0<c \frac{\delta}{1+\delta x} \leq z(x) \leq C \frac{\delta}{1+\delta x}, \quad\left|\partial_{x}^{k} z(x)\right| \leq C \frac{\delta^{k+1}}{(1+\delta x)^{k+1}}, \quad k=1,2 .
$$

Remark 1.2 Note that the condition (1.14) holds when the gas is ideal polytropic at $x=$ $+\infty$.

Our main result in this paper is the following Theorem:

Theorem 1.2 (Stability of transonic stationary solution). Let $\rho_{+}>0, u_{ \pm}<0, \theta_{ \pm}>0$. Assume that $(\hat{\rho}, \hat{u}, \hat{\theta})(x)$ is the solution of the system (1.10) and (1.11) satisfying (1.15) and (1.16) for transonic case $M_{+}=1$, and

$$
\begin{aligned}
& \beta_{1}:=p_{v v}^{+}+\frac{p_{v}^{+}}{v_{+}}>0, \quad \beta_{2}:=\beta_{1} p_{s s}^{+}-\left(p_{v s}^{+}+\frac{p_{s}^{+}}{2 v_{+}}\right)^{2}>0 \\
& \beta_{3}:=-4 v_{+} p_{v}^{+} \beta_{2}-\left(p_{s}^{+}\right)^{2} \beta_{1}>0
\end{aligned}
$$


where $p^{+}=p\left(v_{+}, s_{+}\right)$. Also, suppose that the initial data $\left(\rho_{0}, u_{0}, \theta_{0}\right)$ satisfies

$$
\left(\rho_{0}-\hat{\rho}, u_{0}-\hat{u}, \theta_{0}-\hat{\theta}\right) \in H^{1}(0, \infty), \quad u_{0}(0)=u_{-}, \theta_{0}(0)=\theta_{-} .
$$

Then, there exists a positive constant $\varepsilon_{0}$ such that if

$$
\left\|\left(\rho_{0}-\hat{\rho}, u_{0}-\hat{u}, \theta_{0}-\hat{\theta}\right)\right\|_{1}+\delta \leq \varepsilon_{0}
$$

where $\delta=\left|\left(u_{-}-u_{+}, \theta_{-}-\theta_{+}\right)\right|$, the outflow problem (1.1)-(1.4) has a unique global solution $(\rho, u, \theta)(x, t)$ satisfying

$$
\begin{aligned}
& (\rho-\hat{\rho}, u-\hat{u}, \theta-\hat{\theta}) \in C\left([0, \infty) ; H^{1}(0, \infty)\right), \\
& \rho_{x} \in L_{2}\left(0, \infty ; L_{2}(0, \infty)\right), u_{x}, \theta_{x} \in L_{2}\left(0, \infty ; H^{1}(0, \infty)\right) .
\end{aligned}
$$

Moreover, the solution $(\rho, u, \theta)(x, t)$ convergence to the stationary solution $(\hat{\rho}, \hat{u}, \hat{\theta})(x)$ uniformly as time tends to infinity:

$$
\lim _{t \rightarrow \infty} \sup _{x \in(0, \infty)}|(\rho, u, \theta)(x, t)-(\hat{\rho}, \hat{u}, \hat{\theta})(x)|=0 .
$$

Remark 1.3 The condition (1.18) plays the essential role in the energy estimate for the transonic case (see Lemma 2.1). Note that the condition (1.18) holds when the gas is ideal polytropic at $x=+\infty$. In fact, using (1.5), it is easy to check that

$$
\beta_{1}=\gamma^{2} v_{+}^{-2} p^{+}, \quad \beta_{2}=\frac{(\gamma-1)^{2}}{R^{2}}\left(\gamma-\frac{1}{4}\right) v_{+}^{-2}\left(p^{+}\right)^{2}, \quad \beta_{3}=\frac{(\gamma-1)^{2}}{R^{2}} \gamma(3 \gamma-1) v_{+}^{-2}\left(p^{+}\right)^{3},
$$

for ideal polytropic gas.

The outline of this paper is organized as follows. Section 2 is devoted to Theorem 1.2 . In Appendix, we will give the proof of Proposition 1.1.

\section{Stability of transonic stationary solution}

In this section, we will give the proof of Theorem 1.1. We rewrite (1.1) and (1.10) respectively as

$$
\left\{\begin{array}{l}
\rho_{t}+(\rho u)_{x}=0, \quad x>0, \quad t>0 \\
\rho\left(u_{t}+u u_{x}\right)+p_{x}=\mu u_{x x} \\
\rho\left(e_{t}+u e_{x}\right)+p u_{x}=\kappa \theta_{x x}+\mu u_{x}^{2} \\
\rho \theta\left(s_{t}+u s_{x}\right)=\kappa \theta_{x x}+\mu u_{x}^{2}
\end{array}\right.
$$

and

$$
\left\{\begin{array}{l}
(\hat{\rho} \hat{u})_{x}=0, \quad x>0, \quad t>0, \\
\hat{\rho} \hat{u} \hat{u}_{x}+\hat{p}_{x}=\mu \hat{u}_{x x} \\
\hat{\rho} \hat{u} \hat{e}_{x}+\hat{p} \hat{u}_{x}=\kappa \hat{\theta}_{x x}+\mu \hat{u}_{x}^{2} \\
\hat{\rho} \hat{\theta} \hat{u} \hat{s}_{x}=\kappa \hat{\theta}_{x x}+\mu \hat{u}_{x}^{2}
\end{array}\right.
$$

where $s=s(\rho, \theta)$ denotes the entropy and $\hat{p}=p(\hat{\rho}, \hat{\theta}), \hat{e}=e(\hat{\rho}, \hat{\theta}), \hat{s}=s(\hat{\rho}, \hat{\theta})$. We set the perturbation $(\varphi, \psi, \zeta)(x, t)$ by

$$
(\varphi, \psi, \zeta)(x, t)=(\rho, u, \theta)(x, t)-(\hat{\rho}, \hat{u}, \hat{\theta})(x)
$$


and the solution space $X(I)$ as

$$
X(I)=\left\{(\varphi, \psi, \zeta) \in C\left(I ; H^{1}\right) \mid \varphi_{x} \in L_{2}\left(I ; L_{2}\right),\left(\psi_{x}, \zeta_{x}\right) \in L_{2}\left(I ; H^{1}\right)\right\},
$$

for any interval $I \subset[0, \infty)$. To prove Theorem $[1.2$, it is sufficient to show the following a priori estimate

Proposition 2.1 (A priori estimate) Besides the assumptions of Theorem 1.2, suppose that $(\rho, u, \theta)$ is the solution to the outflow problem (1.1) -(1.4) satisfying $(\phi, \psi, \zeta) \in X([0, T])$. Then, there exists a positive constant $\varepsilon_{1}$ such that if $\sup _{0 \leq t \leq T}\|(\varphi, \psi, \zeta)(t)\|_{1} \leq \varepsilon_{1}$ and $\delta=\left|\left(u_{-}-u_{+}, \theta_{-}-\theta_{+}\right)\right| \leq \varepsilon_{1}$, then for any $t \in[0, T]$, it holds that

$$
\begin{aligned}
\|(\varphi, \psi, \zeta)(t)\|_{1}^{2} & +\int_{0}^{t}\left(\left\|\varphi_{x}\right\|^{2}+\left\|\left(\psi_{x}, \zeta_{x}\right)(\tau)\right\|_{1}^{2}\right) d \tau \\
& +\int_{0}^{t}\left(|\varphi(0, \tau)|^{2}+\left|\varphi_{x}(0, \tau)\right|^{2}\right) d \tau \leq C\|(\varphi, \psi, \zeta)(0)\|_{1}^{2},
\end{aligned}
$$

where $C$ is a positive constant independently of $t, T, \varepsilon_{1}$.

To prove Proposition 2.1, a crucial step is the following energy estimate:

Lemma 2.1 Under the assumptions of Proposition 2.1, it follows that

$$
\begin{aligned}
\|(\varphi, \psi, \zeta)(t)\|^{2} & +\int_{0}^{t}\left\|\left(\psi_{x}, \zeta_{x}\right)(\tau)\right\|^{2} d \tau+\int_{0}^{t}|\varphi(0, \tau)|^{2} d \tau \\
& \leq C\|(\varphi, \psi, \zeta)(0)\|^{2}+C \delta \int_{0}^{t}\left\|\varphi_{x}(\tau)\right\|^{2} d \tau .
\end{aligned}
$$

Proof. Let

$$
\begin{aligned}
\mathcal{E}: & =(e-\hat{e})-\hat{\theta}(s-\hat{s})+\frac{\psi^{2}}{2}+\hat{p}\left(\frac{1}{\rho}-\frac{1}{\hat{\rho}}\right) \\
& =(e-\hat{\theta} s)+\frac{\psi^{2}}{2}+\hat{p}(v-\hat{v})-(\hat{e}-\hat{\theta} \hat{s}),
\end{aligned}
$$

then by (2.1) and (2.2), we have

$$
\begin{aligned}
(\hat{\rho} \mathcal{E})_{t} & +(\hat{\rho} \hat{u} \mathcal{E})_{x}=\hat{\rho} \mathcal{E}_{t}+(\hat{\rho} \hat{u}) \mathcal{E}_{x} \\
& =\frac{\hat{\rho}}{\rho}\left(1-\frac{\hat{\theta}}{\theta}\right)\left(\kappa \theta_{x x}+\mu u_{x}^{2}\right)-\frac{\hat{\rho}}{\rho} p u_{x}-\hat{\rho} \hat{u} s \hat{\theta}_{x}+(\hat{u}-u)\left(\hat{\rho} e_{x}-\hat{\rho} \hat{\theta} s_{x}\right) \\
& +\frac{\hat{\rho}}{\rho} \mu \psi \psi_{x x}+\mu \hat{\rho} \psi \hat{u}_{x x}(v-\hat{v})-\hat{\rho} \psi\left(\frac{p_{x}}{\rho}-\frac{\hat{p}_{x}}{\hat{\rho}}\right)-\hat{\rho} \psi^{2} \hat{u}_{x}-\hat{\rho} \psi^{2} \psi_{x} \\
& +\hat{\rho} \hat{u}\left(\hat{p}_{x}(v-\hat{v})-\hat{p} \hat{v}_{x}\right)+\frac{\hat{\rho}}{\rho} \hat{p} u_{x}+\hat{\rho} \hat{p}(\hat{u}-u) v_{x} \\
& -\hat{\rho} \hat{u}\left(\hat{e}_{x}-\hat{\theta}_{x} \hat{s}-\hat{\theta} \hat{s}_{x}\right)
\end{aligned}
$$

where $\hat{v}=\hat{\rho}^{-1}$. 
Arranging (2.6) yields

$$
\begin{aligned}
(\hat{\rho} \mathcal{E})_{t}+ & (\hat{\rho} \hat{u} \mathcal{E})_{x}=\hat{\rho} \mathcal{E}_{t}+(\hat{\rho} \hat{u}) \mathcal{E}_{x} \\
& =\frac{\hat{\rho}}{\rho}\left(1-\frac{\hat{\theta}}{\theta}\right)\left(\kappa \theta_{x x}+\mu u_{x}^{2}\right) \\
& -\hat{\rho} \hat{u} s \hat{\theta}_{x}+\hat{\rho} \hat{u}\left(\hat{p}_{x}(v-\hat{v})-\hat{p} \hat{v}_{x}\right)-\hat{\rho} \hat{u}\left(\hat{e}_{x}-\hat{\theta}_{x} \hat{s}-\hat{\theta} \hat{s}_{x}\right) \\
& -\frac{\hat{\rho}}{\rho} p u_{x}+\frac{\hat{\rho}}{\rho} \hat{p} u_{x}-\hat{\rho} \psi\left(\frac{p_{x}}{\rho}-\frac{\hat{p}_{x}}{\hat{\rho}}\right) \\
& +\mu \frac{\hat{\rho}}{\rho} \psi \psi_{x x}+\mu \hat{\rho} \psi \hat{u}_{x x}(v-\hat{v})-\hat{\rho} \psi^{2} \hat{u}_{x}-\hat{\rho} \psi^{2} \psi_{x} \\
& +(\hat{u}-u)\left(\hat{\rho} e_{x}-\hat{\rho} \hat{\theta} s_{x}\right)+\hat{\rho} \hat{p}(\hat{u}-u) v_{x} \\
& =I_{1}+I_{2}+I_{3}+I_{4}+I_{5} .
\end{aligned}
$$

Noticing that

$$
\begin{aligned}
\frac{\rho}{\hat{\rho}} I_{1}= & \kappa\left(\frac{\zeta \zeta_{x}}{\theta}\right)_{x}-\kappa \frac{\hat{\theta}}{\theta^{2}} \zeta_{x}^{2}+\kappa \frac{\hat{\theta}_{x} \zeta \zeta_{x}}{\theta^{2}}-\left(\kappa \hat{\theta}_{x x}+\mu \hat{u}_{x}^{2}\right) \frac{\zeta^{2}}{\theta \hat{\theta}} \\
& +\mu \frac{\zeta}{\theta}\left(\psi_{x}^{2}+2 \psi_{x} \hat{u}_{x}\right)+\hat{\rho} \hat{u} \hat{s}_{x}(\theta-\hat{\theta})
\end{aligned}
$$

we have

$$
\begin{aligned}
I_{1}= & \kappa\left(\frac{\hat{\rho} \zeta \zeta_{x}}{\rho \theta}\right)_{x}-\kappa\left(\frac{\varphi}{\rho}\right)_{x} \frac{\zeta \zeta_{x}}{\theta}-\kappa \frac{\hat{\rho} \hat{\theta}}{\rho \theta^{2}} \zeta_{x}^{2}+\kappa \frac{\hat{\rho} \hat{\theta}_{x} \zeta \zeta_{x}}{\rho \theta^{2}} \\
& -\left(\kappa \hat{\theta}_{x x}+\mu \hat{u}_{x}^{2}\right) \frac{\hat{\rho} \zeta^{2}}{\rho \theta \hat{\theta}}+\mu \frac{\hat{\rho} \zeta}{\rho \theta}\left(\psi_{x}^{2}+2 \psi_{x} \hat{u}_{x}\right)+\frac{\hat{\rho}}{\rho} \hat{\rho} \hat{u} \hat{s}_{x}(\theta-\hat{\theta}) .
\end{aligned}
$$

Using $e_{x}=-p v_{x}+\theta s_{x}$, we have

$$
\begin{aligned}
& I_{2}=\hat{\rho} \hat{u}\left(\hat{p}_{x}(v-\hat{v})-\hat{\theta}_{x}(s-\hat{s})\right), \\
& I_{5}=\hat{\rho}(\hat{u}-u)\left((\theta-\hat{\theta}) s_{x}-(p-\hat{p}) v_{x}\right) .
\end{aligned}
$$

Also, we can arrange as follows:

$$
I_{3}=-\left(\frac{\hat{\rho}}{\rho}(p-\hat{p}) \psi\right)_{x}+\left(\frac{\varphi}{\rho}\right)_{x}(p-\hat{p}) \psi-\frac{\hat{\rho}}{\rho}(p-\hat{p}) \hat{u}_{x}-\hat{\rho} \psi \hat{p}_{x}(v-\hat{v})
$$

and

$$
I_{4}=\mu\left(\frac{\hat{\rho}}{\rho} \psi \psi_{x}\right)_{x}-\mu\left(\frac{\varphi}{\rho}\right)_{x} \psi \psi_{x}-\mu \frac{\hat{\rho}}{\rho} \psi_{x}^{2}+\mu \hat{\rho} \psi \hat{u}_{x x}(v-\hat{v})-\hat{\rho} \psi^{2} \hat{u}_{x}-\hat{\rho} \psi^{2} \psi_{x}
$$

Substituting $I_{i}(i=1, \cdots, 5)$ into (2.6) , we have

$$
(\hat{\rho} \mathcal{E})_{t}+(\hat{\rho} \hat{u} \mathcal{E})_{x}+\mu \frac{\hat{\rho} \hat{\theta}}{\rho \theta} \psi_{x}^{2}+\kappa \frac{\hat{\rho} \hat{\theta}}{\rho \theta^{2}} \zeta_{x}^{2}=\Delta_{1 x}+\Delta_{2}+\Delta_{3}+\Delta_{4}
$$


where

$$
\begin{aligned}
\Delta_{1}= & \frac{\mu \hat{\rho} \psi \psi_{x}}{\rho}+\kappa \frac{\hat{\rho} \zeta \zeta_{x}}{\rho \theta}-\frac{\hat{\rho}(p-\hat{p}) \psi}{\rho}-\frac{\hat{\rho} \psi^{3}}{3}, \\
\Delta_{2}= & \kappa \frac{\hat{\rho} \hat{\theta}_{x} \zeta \zeta_{x}}{\rho \theta^{2}}+2 \mu \frac{\hat{\rho} \zeta}{\rho \theta} \psi_{x} \hat{u}_{x}+\mu \hat{\rho} \psi \hat{u}_{x x}(v-\hat{v})+\frac{\hat{\rho}_{x} \psi^{3}}{3}, \\
\Delta_{3}= & -\kappa\left(\frac{\varphi}{\rho}\right)_{x} \frac{\zeta \zeta_{x}}{\theta}+\left(\frac{\varphi}{\rho}\right)_{x}(p-\hat{p}) \psi-\mu\left(\frac{\varphi}{\rho}\right)_{x} \psi \psi_{x}, \\
\Delta_{4}= & -\left(\kappa \hat{\theta}_{x x}+\mu \hat{u}_{x}^{2}\right) \frac{\hat{\rho} \zeta^{2}}{\rho \theta \hat{\theta}}-\hat{\rho} \psi^{2} \hat{u}_{x}-\frac{\hat{\rho}}{\rho}(p-\hat{p}) \hat{u}_{x}-\hat{\rho} \psi \hat{p}_{x}(v-\hat{v}) \\
& +\hat{\rho} \hat{u}\left(\hat{p}_{x}(v-\hat{v})-\hat{\theta}_{x}(s-\hat{s})\right)+\frac{\hat{\rho}}{\rho} \hat{\rho} \hat{u} \hat{s}_{x}(\theta-\hat{\theta}) \\
& +\hat{\rho}(\hat{u}-u)\left((\theta-\hat{\theta}) s_{x}-(p-\hat{p}) v_{x}\right) .
\end{aligned}
$$

It is easy to check that there exist positive constants $c_{i}(i=1,2)$ satisfying

$$
c_{1}\left(\varphi^{2}+\psi^{2}+\zeta^{2}\right) \leq \mathcal{E}(x, t) \leq c_{2}\left(\varphi^{2}+\psi^{2}+\zeta^{2}\right),
$$

due to (1.9) and the assumptions of Proposition 2.1. By using (2.9) and $\left.u\right|_{x=0}=u_{-}<$ $0,\left.(\psi, \zeta)\right|_{x=0}=0$, we have

$$
\left.\Delta_{1}\right|_{x=0}=0, \quad-\left.(\rho u \mathcal{E})\right|_{x=0} \geq c_{3} \varphi^{2}(0, t) .
$$

Integrating (2.8) for $(x, t)$ and using (2.9) and (2.10), we have

$$
\begin{aligned}
\|(\varphi, \psi, \zeta)(t)\|^{2} & +\int_{0}^{t}\left\|\left(\psi_{x}, \zeta_{x}\right)(\tau)\right\|^{2} d \tau+\int_{0}^{t}|\varphi(0, \tau)|^{2} d \tau \\
& \leq C\|(\varphi, \psi, \zeta)(0)\|^{2}+C \sum_{i=2}^{4} \int_{0}^{t} \int_{0}^{\infty}\left|\Delta_{i}\right| d x d \tau
\end{aligned}
$$

By (1.15), we have

$$
\left|\Delta_{2}\right| \leq C \delta\left|\left(\psi_{x}, \zeta_{x}\right)\right|^{2}+C \delta\left(\frac{\delta^{2}}{(1+\delta x)^{4}}+e^{-\hat{c} x}\right)|(\varphi, \psi, \zeta)|^{2}+C \frac{\delta^{2}}{(1+\delta x)^{2}}|\psi|^{3}
$$

and using the inequality

$$
|f(x)| \leq|f(0)|+\sqrt{x}\left\|f_{x}\right\|, \forall f \in H^{1}(0, \infty)
$$

yields

$$
\left.\int_{0}^{\infty}\left|\Delta_{2}\right| d x \leq C \delta \|\left(\varphi_{x}, \psi_{x}, \zeta_{x}\right)\right)\left\|^{2}+C \delta|\varphi(0, t)|^{2}+C \varepsilon_{1} \delta^{\frac{1}{2}}\right\| \psi_{x} \|^{2},
$$

where we used

$$
\begin{gathered}
\int_{0}^{\infty} \frac{\delta^{2}}{(1+\delta x)^{2}}|\psi|^{3} d x \leq C\|\psi\|\left\|\psi_{x}\right\| \int_{0}^{\infty} \frac{\delta^{2}}{(1+\delta x)^{2}}|\psi| d x \\
\quad \leq C\|\psi\|\left\|\psi_{x}\right\|^{2} \int_{0}^{\infty} \frac{\delta^{2} \sqrt{x}}{(1+\delta x)^{2}} d x \leq C \varepsilon_{1} \delta^{\frac{1}{2}}\left\|\psi_{x}\right\|^{2}
\end{gathered}
$$


Noticing that

$$
\left|\Delta_{3}\right| \leq C\left(\left|\varphi_{x}\right|+\left|\hat{\rho}_{x}\right||\varphi|\right)\left(|\zeta|\left|\zeta_{x}\right|+|(\varphi, \psi, \zeta)|^{2}+\left|\psi \| \psi_{x}\right|\right)
$$

and by the argument similar to (2.13), we have

$$
\int_{0}^{\infty}\left|\Delta_{3}\right| d x \leq C\left(\delta+\varepsilon_{1}\right)\left\|\left(\varphi_{x}, \psi_{x}, \zeta_{x}\right)\right\|^{2}+C\left(\delta+\varepsilon_{1}\right)|\varphi(0, t)|^{2} .
$$

It is more difficult to estimate $\Delta_{4}$. We first rewrite $\Delta_{4}$ as

$$
\begin{aligned}
\Delta_{4}= & -(p-\hat{p}) \hat{u}_{x}+\hat{\rho} \hat{u} \hat{p}_{x}(v-\hat{v})-\hat{\rho} \hat{u} \hat{\theta}_{x}(s-\hat{s})+\hat{\rho} \hat{u} \hat{s}_{x}(\theta-\hat{\theta}) \\
& -\left(\kappa \hat{\theta}_{x x}+\mu \hat{u}_{x}^{2}\right) \frac{\hat{\rho} \zeta^{2}}{\rho \theta \hat{\theta}}-\hat{\rho} \psi^{2} \hat{u}_{x} \\
& +\hat{\rho}(\hat{u}-u)\left((\theta-\hat{\theta}) s_{x}-(p-\hat{p}) v_{x}+\hat{p}_{x}(v-\hat{v})\right) \\
& +\left(1-\frac{\hat{\rho}}{\rho}\right)\left(\hat{u}_{x}(p-\hat{p})-\hat{\rho} \hat{u} \hat{s}_{x}(\theta-\hat{\theta})\right)=: \sum_{i=1}^{4} \Delta_{4}^{i} .
\end{aligned}
$$

Using

$$
-\theta_{v}(v, s)=p_{s}(v, s), \quad \hat{p}_{x}=p_{v}(\hat{v}, \hat{s}) \hat{v}_{x}+p_{s}(\hat{v}, \hat{s}) \hat{s}_{x}, \quad \hat{u} \hat{v}_{x}=\hat{u}_{x} \hat{v}
$$

and $\hat{\rho} \hat{\theta} \hat{u} \hat{s}_{x}=\kappa \hat{\theta}_{x x}+\mu \hat{u}_{x}^{2}$ yields

$$
\begin{aligned}
\Delta_{4}^{1}= & -\hat{u}_{x}\left(p-\hat{p}-\hat{p}_{v}(v-\hat{v})-\hat{p}_{s}(s-\hat{s})\right) \\
& +\hat{\rho} \hat{u} \hat{s}_{x}\left(\theta-\hat{\theta}-\hat{\theta}_{v}(v-\hat{v})-\hat{\theta}_{s}(s-\hat{s})\right), \\
\Delta_{4}^{2}= & -\hat{\rho} \psi^{2} \hat{u}_{x}-\frac{\hat{\rho}^{2} \hat{u} \hat{s}_{x}}{\rho \theta} \zeta^{2}, \\
\Delta_{4}^{3}= & \hat{\rho}(\hat{u}-u)\left(\hat{p}_{x}(v-\hat{v})-(p-\hat{p}) \hat{v}_{x}+(\theta-\hat{\theta}) \hat{s}_{x}\right) \\
& +\hat{\rho}(\hat{u}-u)\left((\theta-\hat{\theta})(s-\hat{s})_{x}-(p-\hat{p})(v-\hat{v})_{x}\right),
\end{aligned}
$$

where $\hat{p}_{v}=p_{v}(\hat{v}, \hat{s}), \quad \hat{p}_{s}=p_{s}(\hat{v}, \hat{s}), \quad \hat{\theta}_{v}=\theta_{v}(\hat{v}, \hat{s}), \quad \hat{\theta}_{s}=\theta_{s}(\hat{v}, \hat{s})$. Substituting $\Delta_{4}^{i}(i=$ $1, \cdots, 4)$ into (2.14) and using

$$
\begin{aligned}
& \hat{p}_{x}=p_{v}(\hat{v}, \hat{s}) \hat{v}_{x}+p_{s}(\hat{v}, \hat{s}) \hat{s}_{x}, \\
& p-\hat{p}=p_{v}(\hat{v}, \hat{s})(v-\hat{v})+p_{s}(\hat{v}, \hat{s})(s-\hat{s})+O\left((v-\hat{v})^{2}+(s-\hat{s})^{2}\right),
\end{aligned}
$$

we have

$$
\begin{aligned}
\Delta_{4}= & -\hat{u}_{x}\left(p-\hat{p}-\hat{p}_{v} \phi-\hat{p}_{s} \chi\right)-\hat{\rho} \hat{u}_{x} \psi^{2}-\hat{\rho} \hat{p}_{s} \hat{v}_{x} \psi \chi-\hat{\rho} \hat{p}_{v} \hat{u}_{x} \phi^{2}-\hat{\rho} \hat{p}_{s} \hat{u}_{x} \phi \chi \\
& +\hat{\rho} \hat{u} \hat{s}_{x}\left(\theta-\hat{\theta}-\hat{\theta}_{v}(v-\hat{v})-\hat{\theta}_{s}(s-\hat{s})\right)-\hat{\rho}^{2} \hat{u} \hat{s}_{x}\left(\frac{\zeta^{2}}{\rho u}+\phi \zeta\right) \\
& +\hat{\rho} \psi\left(\zeta \hat{s}_{x}+\hat{p}_{s} \hat{s}_{x} \phi+\zeta \chi_{x}-(p-\hat{p}) \phi_{x}\right)+O\left(\phi^{2}+\chi^{2}\right) \hat{\rho}\left(\psi \hat{v}_{x}+\phi \hat{u}_{x}\right) \\
= & J_{1}+J_{2}+J_{3},
\end{aligned}
$$

where $\phi=v-\hat{v}, \chi=s-\hat{s}$. By the argument similar to (2.13), we have

$$
\left.\int_{0}^{\infty}\left(\left|J_{2}\right|+\left|J_{3}\right|\right) d x \leq C\left(\delta+\varepsilon_{1}\right) \|\left(\varphi_{x}, \psi_{x}, \zeta_{x}\right)\right) \|^{2}+C\left(\delta+\varepsilon_{1}\right)|\varphi(0, t)|^{2}
$$


where we used the fact that $\left|\hat{s}_{x}\right| \leq C\left(\left|\hat{\theta}_{x x}\right|+\left|\hat{u}_{x}\right|^{2}\right) \leq C \frac{\delta^{3}}{(1+\delta x)^{3}}$. By using $\hat{\rho} \hat{u} \hat{v}_{x}=\hat{u}_{x}$ and

$$
p-\hat{p}-\hat{p}_{v} \phi-\hat{p}_{s} \chi=\hat{p}_{v v} \phi^{2}+2 \hat{p}_{v s} \phi \chi+\hat{p}_{s s} \chi^{2}+O\left(\phi^{3}+\chi^{3}\right),
$$

we have

$$
\begin{aligned}
J_{1}= & -\hat{u}_{x}\left(\left(p_{v v}^{+}+\frac{p_{v}^{+}}{v_{+}}\right) \phi^{2}+p_{s s}^{+} \chi^{2}+\rho_{+} \psi^{2}+\left(2 p_{v s}^{+}+\frac{p_{s}^{+}}{v_{+}}\right) \phi \chi+\frac{p_{s}^{+}}{u_{+}} \psi \chi\right) \\
& -\hat{u}_{x}\left(\left(\left(\hat{p}_{v v}-p_{v v}^{+}\right)+\left(\frac{\hat{p}_{v}}{\hat{v}}-\frac{p_{v}^{+}}{v_{+}}\right)\right) \phi^{2}+\left(\hat{p}_{s s}-p_{s s}^{+}\right) \chi^{2}+\left(\hat{\rho}-\rho_{+}\right) \psi^{2}\right) \\
& -\hat{u}_{x}\left(\left(2\left(\hat{p}_{v s}-p_{v s}^{+}\right)+\left(\frac{\hat{p}_{s}}{\hat{v}}-\frac{p_{s}^{+}}{v_{+}}\right)\right) \phi \chi+\left(\frac{\hat{p}_{s}}{\hat{u}}-\frac{p_{s}^{+}}{u_{+}}\right) \psi \chi+O\left(\phi^{3}+\chi^{3}\right)\right) \\
= & :-\sum_{i=1}^{3} J_{1}^{i}
\end{aligned}
$$

where $p^{+}=p\left(v_{+}, s_{+}\right), \hat{p}=p(\hat{v}, \hat{s})$. By the argument similar to (2.13), we have

$$
\left.\int_{0}^{\infty}\left(\left|J_{1}^{2}\right|+\left|J_{1}^{3}\right|\right) d x \leq C\left(\delta+\varepsilon_{1}\right) \|\left(\varphi_{x}, \psi_{x}, \zeta_{x}\right)\right) \|^{2}+C\left(\delta+\varepsilon_{1}\right)|\varphi(0, t)|^{2} .
$$

To estimate $J_{1}^{1}$, we define the quadratic form $f(\phi, \chi, \psi)$ by

$$
f(\phi, \chi, \psi):=\left(p_{v v}^{+}+\frac{p_{v}^{+}}{v_{+}}\right) \phi^{2}+p_{s s}^{+} \chi^{2}+\rho_{+} \psi^{2}+\left(2 p_{v s}^{+}+\frac{p_{s}^{+}}{v_{+}}\right) \phi \chi+\frac{p_{s}^{+}}{u_{+}} \psi \chi .
$$

The matrix $A$ corresponding to the quadratic form $f$

$$
A:=\left(\begin{array}{ccc}
\left(p_{v v}^{+}+\frac{p_{v}^{+}}{v_{+}}\right) & \left(p_{v s}^{+}+\frac{p_{s}^{+}}{2 v_{+}}\right) & 0 \\
\left(p_{v s}^{+}+\frac{p_{s}^{+}}{2 v_{+}}\right) & p_{s s}^{+} & \frac{p_{s}^{+}}{2 u_{+}} \\
0 & \frac{p_{s}^{+}}{2 u_{+}} & \rho_{+}
\end{array}\right),
$$

is positive if and only if all principal minors $\bar{A}_{i}(i=1,2,3)$ of $A$ are positive. Noticing that

$$
M_{+}=1 \rightleftarrows-p_{v}^{+}=\left(\rho_{+} u_{+}\right)^{2},
$$

and by using (1.18), we compute $\bar{A}_{i}(i=1,2,3)$ as follows:

$$
\begin{aligned}
\bar{A}_{1} & =p_{v v}^{+}+\frac{p_{v}^{+}}{v_{+}}>0, \\
\bar{A}_{2} & =\left(p_{v v}^{+}+\frac{p_{v}^{+}}{v_{+}}\right) p_{s s}^{+}-\left(p_{v s}^{+}+\frac{p_{s}^{+}}{2 v_{+}}\right)^{2}>0, \\
\bar{A}_{3} & =\operatorname{det} A=\rho_{+} \bar{A}_{2}-\frac{\left(p_{s}^{+}\right)^{2}}{\left(2 u_{+}\right)^{2}} \bar{A}_{1} \\
& =\frac{1}{\left(2 u_{+}\right)^{2}}\left(-4 v_{+} p_{v}^{+} \bar{A}_{2}-\left(p_{s}^{+}\right)^{2} \bar{A}_{1}\right)>0 .
\end{aligned}
$$


Using (2.18), (1.16) and (1.17), we have

$$
\begin{aligned}
J_{1}^{1}= & -\hat{u}_{x} f(\phi, \chi, \psi) \\
& \leq-c_{4} z(x)^{2}|(\phi, \psi, \chi)|^{2}+C\left(\frac{\delta^{3}}{(1+\delta x)^{3}}+\delta e^{-\hat{c} x}\right)|(\phi, \psi, \chi)|^{2} .
\end{aligned}
$$

Using (2.19), we have by the same lines as in (2.13)

$$
\left.\int_{0}^{\infty}\left(\left|J_{1}^{1}\right|+z(x)^{2}|(\phi, \psi, \chi)|^{2}\right) d x \leq C \delta \|\left(\varphi_{x}, \psi_{x}, \zeta_{x}\right)\right) \|^{2}+C \delta|\varphi(0, t)|^{2} .
$$

By (2.16), (2.17) and (2.20), we have

$$
\left.\int_{0}^{\infty}\left|\Delta_{4}\right| d x \leq C\left(\delta+\varepsilon_{1}\right) \|\left(\varphi_{x}, \psi_{x}, \zeta_{x}\right)\right) \|^{2}+C\left(\delta+\varepsilon_{1}\right)|\varphi(0, t)|^{2} .
$$

Substituting the estimates for $\Delta_{i}(i=2,3,4)$ into (2.11), and choosing $\delta$ to be small, we obtain (2.4). The proof of Lemma 2.1 is completed.

Lemma 2.2 Under the assumptions of Proposition [2.1, it follows that

$$
\left\|\left(\varphi_{x}, \psi_{x}, \zeta_{x}\right)(t)\right\|^{2}+\int_{0}^{t}\left\|\left(\varphi_{x}, \psi_{x x}, \zeta_{x x}\right)(\tau)\right\|^{2} d \tau+\int_{0}^{t}\left|\varphi_{x}(0, \tau)\right|^{2} d \tau \leq C\|(\varphi, \psi, \zeta)(0)\|_{1}^{2} .
$$

Proof. We first estimate $\left\|\varphi_{x}(t)\right\|$. Subtracting the first equation in (2.2) from the first equation in (2.1) and applying $\partial_{x}$ to the resulting equality yields

$$
\varphi_{x t}+u \varphi_{x x}+\rho \psi_{x x}=-2 u_{x} \varphi_{x}-2 \hat{\rho}_{x} \psi_{x}-\hat{u}_{x x} \varphi-\hat{\rho}_{x x} \psi
$$

Also, subtracting the second equation in (2.2) from the second equation in (2.1) yields

$$
\rho \psi_{t}+\rho u \psi_{x}+(p-\hat{p})_{x}=\mu \psi_{x x}+(\hat{u} \hat{\rho}-\rho u) \hat{u}_{x}
$$

We multiply above two equations by $\mu \frac{\varphi_{x}}{\rho^{3}}$ and $\frac{\varphi_{x}}{\rho^{2}}$, respectively, to discover (see [2])

$$
\left(\frac{\mu \varphi_{x}^{2}}{2 \rho^{3}}+\frac{\varphi_{x} \psi}{\rho}\right)_{t}+\left(\frac{\mu u \varphi_{x}^{2}}{2 \rho^{3}}-\frac{\varphi_{t}}{\rho} \psi\right)_{x}+\frac{p_{\rho}(\rho, \theta)}{\rho^{2}} \varphi_{x}^{2}=F_{1},
$$

where

$$
\begin{aligned}
F_{1}:= & -\mu \frac{\varphi_{x}}{\rho^{3}}\left(\hat{\rho}_{x} \psi_{x}+\hat{u}_{x x} \varphi+\hat{\rho}_{x x} \psi\right) \\
& +\frac{\varphi_{x}}{\rho^{2}}(\hat{u} \hat{\rho}-\rho u) \hat{u}_{x}-\frac{u}{\rho} \varphi_{x} \psi_{x}+\frac{(\rho u)_{x} \psi}{\rho^{2}} \hat{\rho}_{x}-\frac{(\rho u)_{x}}{\rho} \psi_{x} \\
& -\frac{\varphi_{x}}{\rho^{2}}\left(p_{\theta}(\rho, \theta) \zeta_{x}+\hat{\rho}_{x}\left(p_{\rho}(\rho, \theta)-p_{\rho}(\hat{\rho}, \hat{\theta})\right)+\hat{\theta}_{x}\left(p_{\theta}(\rho, \theta)-p_{\theta}(\hat{\rho}, \hat{\theta})\right)\right) .
\end{aligned}
$$

By using (1.15), $\left.\psi\right|_{x=0}=0,\left.u\right|_{x=0}=u_{-}<0$ and the assumptions of Proposition 2.1, we have

$$
\begin{aligned}
& \int_{0}^{\infty}\left(\frac{\mu u \varphi_{x}^{2}}{2 \rho^{3}}-\frac{\varphi_{t}}{\rho} \psi\right)_{x} d x=-\frac{\mu u_{-}}{2 \rho^{3}(0, t)} \varphi_{x}^{2}(0, t) \geq c_{5} \varphi_{x}^{2}(0, t), \\
& \int_{0}^{\infty}\left|F_{1}\right| d x \leq C(\delta+\eta)\left\|\varphi_{x}\right\|^{2}+C\left(1+\eta^{-1}\right)\left\|\left(\psi_{x}, \zeta_{x}\right)\right\|^{2}+C \delta|\varphi(0, t)|^{2}
\end{aligned}
$$


for any $\eta>0$. After integrating (2.22) for $(x, t)$, and using (2.23), $p_{\rho}(\rho, \theta)>0$ and (2.4), we have

$$
\left\|\varphi_{x}(t)\right\|^{2}+\int_{0}^{t}\left\|\varphi_{x}(\tau)\right\|^{2} d \tau+\int_{0}^{t}\left|\varphi_{x}(0, \tau)\right|^{2} d \tau \leq C\left\|\left(\varphi, \psi, \zeta, \varphi_{x}\right)(0)\right\|^{2} .
$$

The following, we will estimate $\left\|\left(\psi_{x}, \zeta_{x}\right)\right\|$. Subtracting the second equation in (2.2) from the second equation in (2.1) and multiplying it by $-\frac{\psi_{x x}}{\rho}$ yields

$$
\left(\frac{\psi_{x}^{2}}{2}\right)_{t}-\left(\psi_{t} \psi_{x}\right)_{x}+\frac{\mu \psi_{x x}^{2}}{\rho}=F_{2}
$$

where

$$
F_{2}=u \psi_{x} \psi_{x x}+\frac{(p-\hat{p})_{x}}{\rho} \psi_{x x}+\frac{(\hat{\rho} \hat{u}-\rho u) \hat{u}_{x}}{\rho} \psi_{x x} .
$$

Also, subtracting the third equation in (2.2) from the third equation in (2.1) and using $e_{t}=e_{\theta}(\rho, \theta) \theta_{t}-e_{\rho}(\rho, \theta)(\rho u)_{x}$, we have

$$
\begin{aligned}
\rho e_{\theta}(\rho, \theta) \zeta_{t} & +\rho u \zeta_{x}-\kappa \zeta_{x x}=\rho e_{\rho}(\rho, \theta)(\rho u-\hat{\rho} \hat{u})_{x} \\
& +(\hat{u} \hat{\rho}-\rho u) \hat{e}_{x}-\left(p u_{x}-\hat{p} \hat{u}_{x}\right)+\mu\left(u_{x}^{2}-\hat{u}_{x}^{2}\right) .
\end{aligned}
$$

Multiplying it by $-\frac{\zeta_{x x}}{\rho e_{\theta}(\rho, \theta)}$ yields

$$
\left(\frac{\zeta_{x}^{2}}{2}\right)_{t}-\left(\zeta_{t} \zeta_{x}\right)_{x}+\frac{\kappa \zeta_{x x}^{2}}{\rho e_{\theta}(\rho, \theta)}=F_{3}
$$

where

$$
\begin{aligned}
F_{3} & =\frac{\zeta_{x x}}{\rho e_{\theta}(\rho, \theta)}\left(\rho u \zeta_{x}+\left(p u_{x}-\hat{p} \hat{u}_{x}\right)-\rho e_{\rho}(\rho, \theta)(\rho u-\hat{\rho} \hat{u})_{x}\right) \\
& -\frac{\zeta_{x x}}{\rho e_{\theta}(\rho, \theta)}\left((\hat{u} \hat{\rho}-\rho u) \hat{e}_{x}+\mu\left(u_{x}^{2}-\hat{u}_{x}^{2}\right)\right) .
\end{aligned}
$$

Adding (2.25) and (2.26), we get

$$
\frac{1}{2}\left(\psi_{x}^{2}+\zeta_{x}^{2}\right)_{t}-\left(\psi_{t} \psi_{x}+\zeta_{t} \zeta_{x}\right)_{x}+\left(\frac{\mu \psi_{x x}^{2}}{\rho}+\frac{\kappa \zeta_{x x}^{2}}{\rho e_{\theta}(\rho, \theta)}\right)=F_{2}+F_{3}
$$

By using (1.15) $,\left.\psi\right|_{x=0}=0,\left.u\right|_{x=0}=u_{-}<0$ and the assumptions of Proposition 2.1, we have

$$
\begin{aligned}
& \int_{0}^{\infty}\left(\psi_{t} \psi_{x}+\zeta_{t} \zeta_{x}\right)_{x} d x=0 \\
& \int_{0}^{\infty}\left|F_{2}\right| d x \leq C \eta\left\|\psi_{x x}\right\|^{2}+C\left(\delta+\eta^{-1}\right)\left\|\left(\varphi_{x}, \psi_{x}, \zeta_{x}\right)\right\|^{2}+C \delta|\varphi(0, t)|^{2} \\
& \int_{0}^{\infty}\left|F_{3}\right| d x \leq C \eta\left\|\zeta_{x x}\right\|^{2}+C\left(\delta+\eta^{-1}\right)\left\|\left(\varphi_{x}, \psi_{x}, \zeta_{x}\right)\right\|^{2}+C \delta|\varphi(0, t)|^{2}
\end{aligned}
$$

for any $\eta>0$. After integrating (2.27) for $(x, t)$, and using (2.28), $e_{\theta}(\rho, \theta)>0$, (2.4) and (2.24), we have

$$
\left\|\left(\psi_{x}, \zeta_{x}\right)(t)\right\|^{2}+\int_{0}^{t}\left\|\left(\psi_{x x}, \zeta_{x x}\right)(\tau)\right\|^{2} d \tau \leq C\|(\varphi, \psi, \zeta)(0)\|_{1}^{2} .
$$

By (2.24) and (2.29), we obtain (2.21). The proof of Lemma 2.2 is completed.

On the basis of the above Lemmas 2.1]2.2, we get the Proposition 2.1 at once. Therefore, the Theorem 1.1 is obtained. 


\section{Appendix}

This section is devoted to Proposition 1.1, i.e. we prove the existence of the solution to the stationary problem (1.10) and (1.11). Integrating (1.10) over $[x, \infty)$ yields

$$
\left\{\begin{array}{l}
\hat{\rho} \hat{u}=\rho_{+} u_{+}, x>0, \\
\hat{\rho} \hat{u}^{2}+\hat{p}=\mu \hat{u}_{x}+\rho_{+} u_{+}^{2}+p_{+}, \\
\hat{\rho} \hat{u}\left(\hat{e}+\frac{\hat{u}^{2}}{2}\right)+\hat{p} \hat{u}=\kappa \hat{\theta}_{x}+\mu \hat{u} \hat{u}_{x}+\rho_{+} u_{+}\left(e_{+}+\frac{u_{+}^{2}}{2}\right)+p_{+} u_{+},
\end{array}\right.
$$

where $p_{+}=p\left(v_{+}, \theta_{+}\right), e_{+}=e\left(v_{+}, \theta_{+}\right)$. Integrating the first equation in (1.10) over $[0, x)$ and using (1.11) yields

$$
\hat{\rho} \hat{u}=\rho(0) u_{-}, x>0 .
$$

By (3.1) and (3.2), we have (1.12). So, if $u_{+} \geq 0$, there is no solution to the system (1.10)

and (1.11). Assume that $u_{+}<0$, substituting $\hat{u}=\frac{u_{+}}{v_{+}} \hat{v}\left(v_{+}=\rho_{+}^{-1}, \hat{v}=\hat{\rho}^{-1}\right)$ into $(3.1)_{2}$ and $(3.1)_{3}$, we have

$$
\begin{aligned}
& \hat{v}_{x}=\frac{u_{+}}{\mu v_{+}}\left(\hat{v}-v_{+}\right)+\frac{v_{+}}{\mu u_{+}}\left(p(\hat{v}, \hat{\theta})-p_{+}\right)=: g_{1}(\hat{v}, \hat{\theta}), \\
& \hat{\theta}_{x}=\frac{u_{+}}{\kappa v_{+}}\left(e(\hat{v}, \hat{\theta})-e_{+}\right)-\frac{u_{+}^{3}}{2 \kappa v_{+}^{3}}\left(\hat{v}-v_{+}\right)^{2}+\frac{u_{+}}{\kappa v_{+}} p_{+}\left(\hat{v}-v_{+}\right)=: g_{2}(\hat{v}, \hat{\theta}) .
\end{aligned}
$$

Setting

$$
W=(\hat{v}, \hat{\theta}), G(W)=\left(g_{1}(\hat{v}, \hat{\theta}), g_{2}(\hat{v}, \hat{\theta})\right), W^{-}=\left(v_{b}, \theta_{-}\right), W^{+}=\left(v_{+}, \theta_{+}\right),
$$

we consider the system below

$$
\begin{aligned}
& W_{x}=G(W), \quad x>0 \\
& W(0)=W^{-}, \quad \lim _{x \rightarrow \infty} W(x)=W_{+},
\end{aligned}
$$

where $v_{b}=\frac{v_{+}}{u_{+}} u_{-}$. The Jacobian matrix of $G$ at $W_{+}$is

$$
J_{+}=\left(\begin{array}{cc}
\frac{v_{+}}{\mu u_{+}}\left(\left(\frac{u_{+}}{v_{+}}\right)^{2}+p_{v}\left(v_{+}, \theta_{+}\right)\right) & \frac{v_{+}}{\mu u_{+}} p_{\theta}\left(v_{+}, \theta_{+}\right) \\
\frac{u_{+}}{\kappa v_{+}}\left(e_{v}\left(v_{+}, \theta_{+}\right)+p\left(v_{+}, \theta_{+}\right)\right) & \frac{u_{+}}{\kappa v_{+}} e_{\theta}\left(v_{+}, \theta_{+}\right)
\end{array}\right)
$$

and

$$
\operatorname{det} J_{+}=\frac{1}{\mu \kappa}\left(\frac{u_{+}^{2}}{v_{+}^{2}}+\widetilde{p}_{v}\left(v_{+}, s_{+}\right)\right) e_{\theta}\left(v_{+}, \theta_{+}\right) .
$$

due to (1.6) and (1.7), where $s_{+}=s\left(v_{+}, \theta_{+}\right)$. Using (1.8) and (3.6), we have

$$
\begin{aligned}
M^{+}>1(=1,<1) & \leftrightarrows\left(\frac{u_{+}^{2}}{v_{+}^{2}}+\widetilde{p}_{v}\left(v_{+}, s_{+}\right)\right)>0(=0,<0) \\
& \leftrightarrows \operatorname{det} J_{+}>0(=0,<0)
\end{aligned}
$$

The two eigenvalues $\lambda_{1}, \lambda_{2}$ of $J_{+}$must be satisfied:

$$
\lambda^{2}-b \lambda+\operatorname{det} J_{+}=0
$$


where

$$
b=\frac{v_{+}}{\mu u_{+}}\left(\frac{u_{+}^{2}}{v_{+}^{2}}+\widetilde{p}_{v}\left(v_{+}, s_{+}\right)+\frac{\theta_{+}\left(p_{\theta}\left(v_{+}, \theta_{+}\right)\right)^{2}}{e_{\theta}\left(v_{+}, \theta_{+}\right)}\right)+\frac{u_{+}}{\kappa v_{+}} e_{\theta}\left(v_{+}, \theta_{+}\right) .
$$

By using (3.7)-(3.9), (1.2) and $u_{+}<0$, we have

$$
\begin{aligned}
& \text { if } M^{+}>1, \text { then } b<0 \text { and } \lambda_{1}<\lambda_{2}<0, \\
& \text { if } M^{+}<1, \text { then } \lambda_{1}<0<\lambda_{2},
\end{aligned}
$$

where we used

$$
b^{2}-4 \operatorname{det} J_{+} \geq \frac{4 u_{+}^{2}}{\mu \kappa v_{+}^{2}} \theta_{+}\left(p_{\theta}\left(v_{+}, \theta_{+}\right)\right)^{2}>0 .
$$

Thus, it is easy to prove the case 1) and 2) in Proposition 1.1 by the same lines as in [11. We prove the case 3) in Proposition 1.1. Due to (3.5)-(3.7), for transonic case $M_{+}=1$, we have

$$
J_{+}=\left(\begin{array}{cc}
\frac{v_{+}}{\mu u_{+}} \frac{\theta_{+}\left(p_{\theta}^{+}\right)^{2}}{e_{\theta}^{+}} & \frac{v_{+}}{\mu u_{+}} p_{\theta}^{+} \\
\frac{u_{+}}{\kappa v_{+}} \theta_{+} p_{\theta}^{+} & \frac{u_{+}}{\kappa v_{+}} e_{\theta}^{+}
\end{array}\right) \equiv\left(\begin{array}{cc}
a_{11} & a_{12} \\
a_{21} & a_{22}
\end{array}\right), \operatorname{det} J_{+}=0 .
$$

and the eigenvalues of $J_{+}$are $\lambda_{1}=0$ and $\lambda_{2}=a_{11}+a_{22}<0$ of which corresponding eigenvectors are given by

$$
r_{1}=\left(\begin{array}{c}
1 \\
-\frac{a_{11}}{a_{12}}
\end{array}\right) \equiv\left(\begin{array}{c}
1 \\
-\frac{\theta_{+} p_{\theta}^{+}}{e_{\theta}^{+}}
\end{array}\right), \quad r_{2}=\left(\begin{array}{c}
\frac{a_{11}}{a_{12}} \\
\frac{a_{21}}{a_{12}}
\end{array}\right) \equiv\left(\begin{array}{c}
\frac{\theta_{+} p_{\theta}^{+}}{e_{\theta}^{+}} \\
\frac{\mu \theta_{+} u_{+}^{2}}{\kappa v_{+}^{2}}
\end{array}\right),
$$

where $p_{\theta}^{+}=p_{\theta}\left(v_{+}, \theta_{+}\right), e_{\theta}^{+}=e_{\theta}\left(v_{+}, \theta_{+}\right)$. Defining the matrix $B$ by

$$
B=\left(\begin{array}{cc}
1 & \frac{\theta_{+} p_{\theta}^{+}}{e_{\theta}^{+}} \\
-\frac{\theta_{+} p_{\theta}^{+}}{e_{\theta}^{+}} & \frac{\mu \theta_{+} u_{+}^{2}}{\kappa v_{+}^{2}}
\end{array}\right) \equiv\left(\begin{array}{cc}
1 & b_{1} \\
-b_{1} & b_{2}
\end{array}\right)
$$

we have

$$
B^{-1} J_{+} B=\left(\begin{array}{cc}
0 & 0 \\
0 & \lambda_{2}
\end{array}\right)=: \Lambda, \quad B^{-1}=\frac{1}{b_{2}+b_{1}^{2}}\left(\begin{array}{cc}
b_{2} & -b_{1} \\
b_{1} & 1
\end{array}\right) .
$$

Setting $Y \equiv\left(y_{1}, y_{2}\right)^{*}:=B^{-1}\left(W-W^{+}\right)^{*}$, we rewrite (3.4) as

$$
\begin{aligned}
& Y_{x}=\Lambda Y+B^{-1}\left(G(W)-J_{+}\left(W-W^{+}\right)\right)=: \Lambda Y+H(Y), \quad x>0, \\
& Y(0)=Y_{0} \equiv B^{-1}\left(W^{-}-W^{+}\right)^{*}, \quad \lim _{x \rightarrow \infty} Y(x)=0,
\end{aligned}
$$

where

$$
H(Y)=B^{-1}\left(\begin{array}{c}
\frac{v_{+}}{\mu u_{+}}\left(p(\hat{v}, \hat{\theta})-p^{+}-p_{v}^{+}\left(\hat{v}-v_{+}\right)-p_{\theta}^{+}\left(\hat{\theta}-\theta_{+}\right)\right) \\
\frac{u_{+}}{\kappa v_{+}}\left(e(\hat{v}, \hat{\theta})-e^{+}-e_{v}^{+}\left(\hat{v}-v_{+}\right)-e_{\theta}^{+}\left(\hat{\theta}-\theta_{+}\right)\right)-\frac{u_{+}^{3}}{2 \kappa v_{+}^{3}}\left(\hat{v}-v_{+}\right)^{2}
\end{array}\right) .
$$

Let's denote $\left(\tilde{f}_{1}, \tilde{f}_{2}\right)=B H(Y)$. Using (3.16) and

$$
\hat{v}-v_{+}=y_{1}+b_{1} y_{2}, \hat{\theta}-\theta_{+}=-b_{1} y_{1}+b_{2} y_{2},
$$


we have

$$
\begin{aligned}
\tilde{f}_{1}\left(y_{1}, y_{2}\right)= & \frac{v_{+}}{\mu u_{+}}\left(p_{v v}^{+}\left(\hat{v}-v_{+}\right)^{2}+2 p_{v \theta}^{+}\left(\hat{v}-v_{+}\right)\left(\hat{\theta}-\theta_{+}\right)+p_{\theta \theta}^{+}\left(\hat{\theta}-\theta_{+}\right)^{2}\right) \\
& +O\left(\left|\left(\hat{v}-v_{+}, \hat{\theta}-\theta_{+}\right)\right|^{3}\right) \\
= & \frac{v_{+}}{\mu u_{+}}\left(p_{v v}^{+}-2 b_{1} p_{v \theta}^{+}+b_{1}^{2} p_{\theta \theta}^{+}\right) y_{1}^{2}+O\left(\left|y_{1}\right|^{3}+\left|y_{1}\right|\left|y_{2}\right|+\left|y_{2}\right|^{2}\right), \\
\tilde{f}_{2}\left(y_{1}, y_{2}\right)= & \frac{u_{+}}{\kappa v_{+}}\left(e_{v v}^{+}\left(\hat{v}-v_{+}\right)^{2}+2 e_{v \theta}^{+}\left(\hat{v}-v_{+}\right)\left(\hat{\theta}-\theta_{+}\right)+e_{\theta \theta}^{+}\left(\hat{\theta}-\theta_{+}\right)^{2}\right) \\
& -\frac{u_{+}^{3}}{2 \kappa v_{+}^{3}}\left(\hat{v}-v_{+}\right)^{2}+O\left(\left|\left(\hat{v}-v_{+}, \hat{\theta}-\theta_{+}\right)\right|^{3}\right) \\
= & \frac{u_{+}}{\kappa v_{+}}\left(e_{v v}^{+}-2 b_{1} e_{v \theta}^{+}+b_{1}^{2} e_{\theta \theta}^{+}-\frac{u_{+}^{2}}{2 v_{+}^{2}}\right) y_{1}^{2}+O\left(\left|y_{1}\right|^{3}+\left|y_{1}\right|\left|y_{2}\right|+\left|y_{2}\right|^{2}\right),
\end{aligned}
$$

where $p_{v v}^{+}=p_{v v}\left(v_{+}, \theta_{+}\right), e_{v \theta}^{+}=e_{v \theta}\left(v_{+}, \theta_{+}\right)$and so on. By (3.14), (3.15), (3.18) and (3.19), we have

$$
\left\{\begin{array}{l}
y_{1 x}=-a_{+} y_{1}^{2}+f_{1}\left(y_{1}, y_{2}\right), \\
y_{2 x}=\lambda_{2} y_{2}+f_{2}\left(y_{1}, y_{2}\right), \quad Y=\left(y_{1}, y_{2}\right)^{*} \\
Y(0)=Y_{0}, \quad \lim _{x \rightarrow \infty} Y(x)=0
\end{array}\right.
$$

where

$$
\begin{aligned}
& a_{+}=-\frac{v_{+} b_{2}}{\mu u_{+}\left(b_{2}+b_{1}^{2}\right)}\left(p_{v v}^{+}-2 b_{1} p_{v \theta}^{+}+b_{1}^{2} p_{\theta \theta}^{+}\right) \\
& \quad+\frac{u_{+} b_{1}}{\kappa v_{+}\left(b_{2}+b_{1}^{2}\right)}\left(e_{v v}^{+}-2 b_{1} e_{v \theta}^{+}+b_{1}^{2} e_{\theta \theta}^{+}-\frac{u_{+}^{2}}{2 v_{+}^{2}}\right), \\
& f_{1}\left(y_{1}, y_{2}\right)=\left(b_{2}+b_{1}^{2}\right)^{-1}\left(b_{2} \tilde{f}_{1}-b_{1} \tilde{f}_{2}\right)+a_{+} y_{1}^{2}=O\left(\left|y_{1}\right|^{3}+\left|y_{1}\right|\left|y_{2}\right|+\left|y_{2}\right|^{2}\right), \\
& f_{2}\left(y_{1}, y_{2}\right)=\left(b_{2}+b_{1}^{2}\right)^{-1}\left(b_{1} \tilde{f}_{1}+\tilde{f}_{2}\right)=O\left(\left|y_{1}\right|^{2}+\left|y_{1}\right|\left|y_{2}\right|+\left|y_{2}\right|^{2}\right) .
\end{aligned}
$$

By (3.13), (1.2), $u_{+}<0, e_{v \theta}(v, \theta)=\theta p_{\theta \theta}(v, \theta)$ and (1.14), we have $a_{+}>0$. Therefore, applying the center manifold theory(see [1]), by the same lines as in subsection 2.1 of [1], there exist a local center manifold $y_{2}=h^{c}\left(y_{1}\right)$ and a local stable manifold $y_{1}=h^{s}\left(y_{2}\right)$ such that if the data $\left(y_{01}, y_{02}\right)$ satisfies

$$
y_{02}=h^{c}\left(y_{01}\right) \quad \text { and } \quad y_{01} \geq h^{s}\left(y_{02}\right),
$$

then the problem (3.20) has a unique smooth solution satisfying

$$
\left|\partial_{x}^{k} Y(x)\right| \leq \frac{C \sigma^{k+1}}{(1+\sigma x)^{k+1}}+C \sigma \exp (-c x), \quad k=0,1,2,
$$

where $\sigma=\left|Y_{0}\right|$ and $C$ is a positive constant independently of $x, \sigma$. Moreover, we have

$$
\begin{aligned}
& y_{1 x}=-a_{+} z^{2}(x)+O\left(z^{3}(x)+\sigma \exp (-c x)\right), \\
& y_{2 x}=O\left(z^{3}(x)+\sigma \exp (-c x)\right) .
\end{aligned}
$$

where $z(x)$ is given as a solution to the equation

$$
z_{x}=-a_{+} z^{2}+f_{1}\left(z, h^{c}(z)\right)
$$


and satisfies

$$
0<c \frac{\sigma}{1+\sigma x} \leq z(x) \leq C \frac{\sigma}{1+\sigma x}, \quad\left|\partial_{x}^{k} z(x)\right| \leq C \frac{\sigma^{k}}{(1+\sigma x)^{k}}, \quad k=1,2 .
$$

Using (3.17), (3.22),$v_{+}>0$ and $\theta_{+}>0$, it is easy to check that there is a positive constant $\epsilon_{0}$ such that if $\sigma=\left|Y_{0}\right| \leq \epsilon_{0}$, then

$$
\underline{m} \leq \hat{v}(x), \hat{\theta}(x) \leq \bar{m},
$$

where $\underline{m}$ and $\bar{m}$ are positive constants independently of $x$. By (3.17), (3.22), (3.23), (3.25), (1.12) and $u_{+}<0$, we get (1.15) and (1.16). The proof of Proposition 1.1 is completed.

Acknowledgments: The authors would like to thank the referees for their valuable comments. The corresponding author Xiaoding Shi and other two authors were partially supported by National Natural Sciences Foundation of China No. 11671027, 11471321 and 11371348.

\section{References}

[1] J. Caar, Applications of Center Manifold Theory, Springer-Verlag, 1981.

[2] Y. Chen, H. Hong, X. Shi, Stability and convergence rate of stationary solutions to outflow problem for full Navier-Stokes equations of general gas, Submitted to Appl. Anal., (2017).

[3] L. Fan, H. Liu, T. Wang, H. J. Zhao, Inflow problem for the one-dimensional compressible Navier-Stokes equations under large initial perturbation, J. Diff. Equs., 257 (10) (2014) 3521-3553.

[4] H. Hong, T. Wang, Stability of stationary solutions to the inflow problem for full compressible Navier-Stokes equations with a large initial perturbation, SIAM J. Math. Anal., 49 (3) (2017) 2138-2166.

[5] H. Hong, T. Wang, Large-time behavior of solutions to the inflow problem of full compressible Navier-Stokes equations with large perturbation, Nonlinearity, 30 (8) (2017) 3010-3039.

[6] F. M. Huang, J. Li, X. Shi, Asymptotic behavior of solutions to the full compressible Navier-Stokes equations in the half space, Commun. Math. Sci., 8 (2010) 639-654.

[7] F. M. Huang, A. Matsumura, X. Shi, Viscous shock wave and boundary layer solution to an inflow problem for compressible viscous gas. Comm. Math. Phys., 239 (2003) 261-285.

[8] F. M. Huang, X. Qin, Stability of boundary layer and rarefaction wave to an outflow problem for compressible Navier-Stokes equations under large perturbation, J. Diff. Equs., 246 (2009) 4077-4096.

[9] Y. Kagei, S. Kawashima, Syability of planar stationary solution to the compressible Navier-Stokes equation on the half space, Commun. Math. Phys., 266 (2006) 401-430. 
[10] S. Kawashima, A. Matsumura, Asymptotic stability of travelling waves solutions of systems for one-dimensional gas motion, Commun. Math. Phys., 101 (1985) 97-127.

[11] S. Kawashima, T. Nakamura, S. Nishibata, P. Zhu, Stationary waves to viscous heatconductive gases in the half space: existence, stability and convergence rate, Math. Models mech. Appl. Sci., 20 (12) (2010) 2201-2235.

[12] S. Kawashima, S. Nishibata, P. Zhu, Asymptotic stability of the stationary solution to the compressible Navier-Stokes equations in the half space, Comm. Math. Phys., 240 (2003) 483-500.

[13] S. Kawashima, P. Zhu, Asymptotic stability of nonlinear wave for the compressible Navier-Stokes equations in the half space, J. Diff. Equs., 244 (2008) 3151-3179.

[14] S. Kawashima, P. Zhu, Asymptotic stability of rarefaction wave for the Navier-Stokes equations for a compressible fluid in the half space, Arch. Rational Mech. Anal., 194 (2009) 105-132.

[15] A. Matsumura, Inflow and outflow problems in the half space for a one-dimensional isentropic model system of compressible viscous gas, in: IMS Conference on Differential Equations from Mechanics, Hong Kong, 1999, Methods Appl. Anal., 8 (4) (2001) 645666.

[16] A. Matsumura, M. Mei, Convergence to traveling fronts of solutions of the p-system with viscosity in the presence of a boundary, Arch. Rat. Mech. Anal., 146 (1999) 1-22.

[17] A. Matsumura, K. Nishihara, Global asymptotics towards the rarefaction waves for the solutions of viscous p-system with boundary effect, Quart. Appl. Math., 58 (2000) 69-83.

[18] A. Matsumura, K. Nishihara, Large-time behavior of solutions to an inflow problem in the half space for a one-dimensional system of compressible viscous gas. Comm. Math. Phys., 222 (2001) 449-474.

[19] T. Nakamura, S. Nishibata, Convergence rate toward planar stationary waves for compressible viscous fluid in multididmensional half line, SIAM J. Math. Anal., 41 (5) (2009) 1757-1791.

[20] T. Nakamura, S. Nishibata, Stationary wave associated with an inflow problem in the half line for viscous heat-conductive gas, J. Hyper. Diff. Equs., 8 (4) (2011) 657-670.

[21] T. Nakamura, S. Nishibata, T. Yuge, Convergence rate of solutions toward stationary solutions to the compressible Navier-Stokes equation in a half line, J. Diff. Equs., 241 (2007) 94-111.

[22] T. Nakamura, Y. Ueda, S. Kawashima, Convergence rate toward degenerate stationary wave for compressible viscous gases, Proc. Internat. Conf. on Nonlinear Analysis and Convex Analysis (2009) 239-248.

[23] X. Qin, Large-time behaviour of solutions to the outfow problem of full compressible Navier-Stokes equations, Nonlinearity, 24 (2011) 1369-1394. 
[24] X. Qin, Y. Wang, Stability of wave patterns to the inflow problem of full compressible Navier-Stokes equations, SIAM J. Math. Anal., 41 (2009) 2057-2087.

[25] X. Qin, Y. Wang, Large-time behavior of solutions to the inflow problem of full compressible Navier-Stokes equations, SIAM J. Math. Anal., 43 (2011) 341-346.

[26] X. Shi, On the stability of rarefaction wave solutions for viscous p-system with boundary effect, Acta Math. Applicatae Sinica, English Series, 19 (2) (2003) 341-352.

[27] J. Smoller, Shock waves and reaction-diffusion equations, Springer-Verlag, New York, second eddition, 1994.

[28] L. Wan, T. Wang, H. J. Zhao, Asymprotic stability of wave patterns to compressible viscous and heat-conducting gases in the half space, arXiv:1506.07626v1 [math.Ap] 25 Jun 2015.

[29] L. Wan, T. Wang, Q. Zou, Stability of stationary solutions to the outflow problem for full compressible Navier-Stokes equations with large initial perturbation, Nonlinearity, 29 (2016) 1329-1354.

[30] T. Zheng, J. Zhang, J. Zhao, Asymptotic stability of viscous contact discontinuinity to an inflow problem for compressible Navier-Stokes equations, Nonlinear Analysis, T. M. A., 74 (2011) 6617-6639. 